\title{
Dual-domain mixing cell modelling and uncertainty analysis for unsaturated bromide and chloride transport
}

\author{
Th. Wöhling $^{\text {a,b }}$, G. F. Barkle ${ }^{\text {c }}$, V. J. Bidwell ${ }^{\text {a }}$, R. Dann ${ }^{\text {d }}$, A. Wall a ${ }^{\text {, B. Moorhead }}{ }^{\text {a }}$, \\ J. Clague ${ }^{a}$, and J. A. Vrugt ${ }^{\mathrm{e}}$ \\ ${ }^{a}$ Lincoln Ventures (LVL), Private Bag 3062, Hamilton, New Zealand \\ Email: thomas.woehling@lvl.co.nz \\ ${ }^{b}$ Water \& Earth System Science (WESS), Univ. of Tübingen, Hölderlinstr. 12, 72074 Tübingen, Germany \\ ${ }^{c}$ Aqualinc Research Ltd, PO Box 14-041, Hamilton, New Zealand \\ ${ }^{d}$ Institute of Environmental Science and Research (ESR), PO Box 29-181, Christchurch, New Zealand \\ ${ }^{e}$ Dept. of Civil and Environmental Eng., Univ. of California, Irvine, Irvine, 92697 CA, USA
}

\begin{abstract}
Land use intensification is considered the main reason for early signs of deterioration in the water quality of Lake Taupo, New Zealand. Little is understood, however about the origin, and governing flow paths of the contaminants and their respective transformation processes that affect the water quality of Lake Taupo. In this study we investigate contaminant transport and its small-scale variability in the volcanic vadose zone surrounding the Lake. Lateral and preferential solute transport is analysed to better understand the risks of diffuse groundwater pollution from con-
\end{abstract} taminant sources at the land surface.

As part of the investigations into this problem the Spydia experimental facility has been installed under a pastoral agriculture land use in the Lake Taupo region, New Zealand (Barkle et al. 2011). A multiple tracer experiment was conducted at the site and vadose zone drainage volumes were measured using Automated Equilibrium Tension Plate Lysimeters (Figure 1). The chemical composition of the drainage samples was analysed in the laboratory.

A dual-domain mixing cell model was set up to simulate the unsaturated advective-dispersive tracer transport at selected monitoring sites for two different bromide-chloride $\left(\mathrm{Br}^{-}, \mathrm{Cl}^{-}\right)$tracers that were applied at the land surface at two different regions (Figure 1). Some model parameters were constrained by mixing calculations of the measured total $\mathrm{Br}^{-}$and $\mathrm{Cl}^{-}$load, whereas others were calibrated using the measured $\mathrm{Br}^{-}$and $\mathrm{Cl}^{-}$breakthrough curves and drainage volumes. Multi-objective inverse modelling using the AMALGAM evolutionary search method (Vrugt \& Robinson, 2007) showed a significant trade-off between simulated transient $\mathrm{Br}^{-}$and $\mathrm{Cl}^{-}$breakthrough curves and corresponding drainage volumes, but also a compromise solution that fits both objective functions reasonably well.

Estimates of parameter and model predictive uncertainty were subsequently derived using the differen-

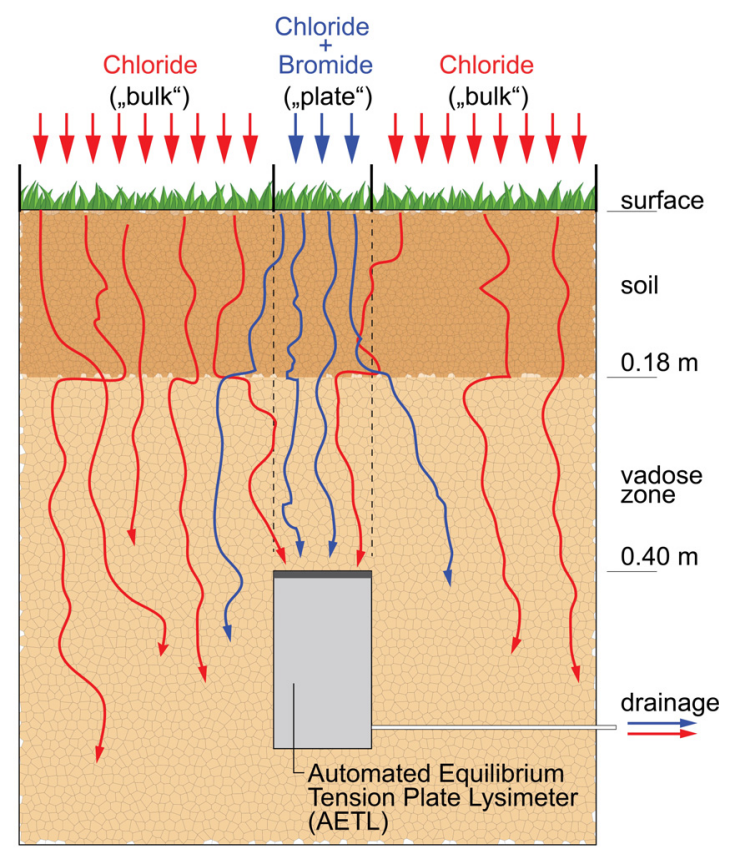

Figure 1. Multiple tracer experiment with different chloride and bromide compartments and potential flow paths of the plate (blue) and bulk tracer (red) from the soil surface to the Automated Equilibrium Tension Plate Lysimeter (AETL). tial evolution adaptive metropolis, DREAM $\mathrm{ZS}_{\mathrm{ZS}}$ adaptive Markov chain Monte Carlo algorithm (Vrugt et al., 2011) with a formal Bayesian likelihood function (Wöhling \& Vrugt, 2011). Uncertainty bounds derived by this MCMC method simultaneously capture the observed $\mathrm{Br}^{-}$and $\mathrm{Cl}^{-}$breakthrough curves and corresponding drainage volumes. Our results demonstrate that (1) flow and transport in the vadose zone is highly variable, and (2) contaminants at the land surface can travel rapidly through the soil to larger depths and this cannot be described with the classical advection-dispersion equation.

Keywords: Mixing cell model, dual-domain contaminant transport, uncertainty assessment, Pareto analysis 


\section{INTRODUCTION}

Non-point sources are the main causes for groundwater and surface water pollution in New Zealand. About half the lengths of New Zealand's rivers are located in agricultural catchments and generally have much poorer water quality than water bodies in undeveloped catchments (MfE, 2007). It is predicted that nutrient levels in rivers and streams will increase further. Therefore, understanding and minimising non-point source contamination is vital to the sustainable development of primary production and the protection of groundwater and surface water resources. In this study we aim at a better understanding of diffuse contaminant transport from the land surface into the unsaturated zone under field conditions.

One-dimensional advective-dispersive solute transport with variable drainage intervals can be simulated with a finite volume mixing cell approach with pore water drainage discretization (Bidwell, 2000). Complete solute mixing is assumed within each cell. Interaction with an immobile phase and transformations such as degradation can also be simulated (Bidwell, 1999). Dann et al. (2010) used two noninteracting mixing-cell models to simulate advective-dispersive and macropore transport in gravel.

We modified the mixing cell model to simulate dual-domain solute tracer transport originating from different flow regions in the Spydia vadose zone. The model was set up to simulate the contributions of two different tracers that were applied at two different regions at the soil surface (Figure 1). Field experiments conducted at the site show that water flow and solute transport in the vadose zone is spatially and temporally variable and that preferential transport processes may be causing this variability at some locations. Another source of this variability is the different extent of the capture zone of leachate sampling devices. Even when capture efficiencies are close to $100 \%$, which is rarely seen in field studies, the collected leachate originates not necessarily from predominantly vertical transport (as indicated by idealized flow paths in Figure 1). With our proposed modelling approach, we explore capture zones and differences in the predominant unsaturated flow and transport processes at two comparable locations in the vadose zone. Adequate levels of model complexity are tested for these locations by adapting the number of parallel mixing cell models and by separating or lumping the parameters for the two model domains.

The model parameters are calibrated and the trade-off between simulated tracer breakthrough curves (BTC) and drainage volumes is analysed using field experimental data and the multi-objective evolutionary search algorithm AMALGAM (Vrugt und Robinson, 2007). Parameter and model predictive uncertainty is estimated using the differential evolution adaptive metropolis, DREAM $_{\mathrm{ZS}}$ adaptive Markov chain Monte Carlo scheme (Vrug et al., 2011; Wöhling \& Vrugt, 2011).

\section{MATERIALS AND METHODS}

\subsection{Field experiments}

The experimental data originates from the Spydia experimental site located in the Tutaeuaua subcatchment (Landcorp's Waihora Sta-

Table 1. Chemical composition of the applied plate and bulk tracers

tion, $175^{\circ} 74.997^{\prime}$ E, $38^{\circ} 36.863^{\prime}$ S) north of Lake Taupo, New Zealand. The site was established on a sheep and beef farm under pastoral land use. The vadose zone materials at Spydia encompass a young volcanic soil (active root zone $0-0.18 \mathrm{~m}$ depth) which belongs to the Oruanui loamy sand series that developed on the underlying unwelded Taupo Ignimbrite.

Automated Equilibrium Tension Plate Lysimeters (AETLs) with a $0.198 \mathrm{~m}^{2}$ sampling area were installed at different depths in the Spydia vadose zone to monitor water and solute fluxes in the unsaturated zone. In this study we will focus on AETL1 and AETL3 that both are installed at the $0.4 \mathrm{~m}$ depth around a central access caisson with $2.3 \mathrm{~m}$ diameter. Rainfall is measured on site and aggregated to daily values for use in our modelling approach. We neglect evapotranspiration during the experiment (conducted during winter) and assume that daily drainage values equal daily rainfall values. Details about the experimental facility are reported in Wöhling et al. (2009) and Barkle et al. (2011) and are therefore not repeated herein.

Two tracers with different $\mathrm{Br}^{-}$and $\mathrm{Cl}^{-}$composition were applied at the soil surface above all AETLs at the site on August 08, 2010. To differentiate leachate originating from vertical transport and leachate from other regions, we applied a "plate tracer" vertically above the sampling area of the individual AETLs and a "bulk tracer" at the remaining area around the circumference of the access caisson (Figure 1). The sections at the surface directly above the AETLs were identified using ground penetrating radar (GPR) and separated using tailor-made steel segments. Both the plate and the bulk tracer were applied at an area weighted volume of 10 $\mathrm{mm}$. The application time was about 30 minutes for all AETLs. Before and after tracer application, an area weighted volume of $2 \mathrm{~mm}$ water was applied to enhance tracer penetration into the subsurface. The chemical 
composition of the plate and bulk tracer application is summarized in Table 1. During the 80 days following application, the tracer was transported in the vadose zone under natural occurring climatic conditions. The $\mathrm{Br}^{-}$and $\mathrm{Cl}^{-}$concentrations in the leachate collected by the AETLs during this time period was analysed in the laboratory.

\subsection{Differentiating tracer sources}

The mass of bromide and chloride captured by the individual AETLs can be estimated by:

$$
\begin{aligned}
& M_{\text {Bromide }}=C_{\text {bulk }, \mathrm{Br}} \cdot V_{\text {bulk }}+C_{\text {plate }, \mathrm{Br}} \cdot V_{\text {plate }} \\
& M_{\text {Chloride }}=C_{\text {bulk }, \mathrm{Cl}} \cdot V_{\text {bulk }}+C_{\text {plate, } \mathrm{Cl}} \cdot V_{\text {plate }}
\end{aligned}
$$

where $M_{\text {Bromide }}\left(M_{\text {Chloride }}\right)$ is the total $\mathrm{Br}^{-}\left(\mathrm{Cl}^{-}\right)$mass collected by the AETLs [mg] as derived from the chemical analysis of the leachate. $C_{b u l k, B r}\left(C_{b u l k, C l}\right)$ and $C_{\text {plate, } B r}\left(C_{\text {plate, } C l}\right)$ denote the concentrations of bromide (chloride) in the bulk and plate tracer [mg/l], respectively. $V_{\text {bulk }}$ and $V_{\text {plate }}$ are the drainage volumes originating from the bulk and plate regions. Inserting the $\mathrm{Br}^{-}$and $\mathrm{Cl}^{-}$tracer concentrations in (1) and reordering the equations, yields the total drainage volume originating from the bulk and plate regions:

$$
\begin{aligned}
& V_{\text {bulk }}=1.038 \cdot\left[\frac{M_{\text {Chloride }}}{4400 \mathrm{mg} / 1}-\frac{M_{\text {Bromide }}}{5200 \mathrm{mg} / 1}\right] \\
& V_{\text {plate }}=2.89 \mathrm{E}^{-3} \cdot\left[\frac{M_{\text {Bromide }}}{15 \mathrm{mg} / 1}-\frac{M_{\text {Chloride }}}{4250 \mathrm{mg} / 1}\right] .
\end{aligned}
$$

The percentage of captured tracer volume from the plate and bulk areas, $v_{p}$ and $v_{b}$, can be calculated by

$$
v_{p}=V_{\text {plate }} / V_{\text {app }} \cdot 100 \% ; \quad v_{b}=V_{\text {bulk }} / V_{\text {app }} \cdot 100 \%
$$

where $V_{a p p}$ is the applied tracer volume (1.98 1) relative to the surface area of the AETL.

\subsection{Dual-region, dual-domain mixing cell model}

We utilize up to four different one-dimensional mixing cell models in parallel to simulate advectivedispersive solute transport in the soil matrix and the preferential flow domains of the two different flow regions (the plate and the bulk tracer regions). In a single mixing cell model, the concentration in the $r$-th cell ( $r$ $=1,2 \ldots n$ ) after the $k$-th drainage interval, $d I(k)$, is given by (Bidwell, 2000):

$$
c_{r}(k)=\exp [-\alpha(k)] \cdot\left[\sum_{m=0}^{r-1}\left(b_{m}(k) c_{r-m}(k-1)\right)\right]+\left[1-\exp [-a(k)] \cdot \sum_{m=0}^{r-1}\left(b_{m}(k)\right)\right] \cdot c_{i n}(k),
$$

where,

$$
\alpha(k)=\frac{d I(k)}{2 \lambda \theta} \quad ; \quad b_{m}(k)=\frac{\alpha(k)^{m}}{m !}
$$

in which $c_{i n}(k)$ denotes the mean inflow concentration to the first cell, $\lambda$ signifies the dispersivity, and $\theta$ represents the transporting volume fraction. No retardation of solutes is considered in this study. The Peclet number is given as $P e=L / \lambda$, where $L$ is the characteristic length. Each mixing cell has a length of $2 \lambda$, so that the number of mixing cells, $n=L / 2 \lambda=P e / 2$. Equation (5) is used to calculate the resident concentration in the mixing cells of the different parallel models. The corresponding solute fractions are combined to calculate the total solute concentration, $c_{\text {total,out }}$, collected by the AETL:

$$
c_{\text {total }, \text { out }}(k)=\omega_{b} v_{b} c_{n}^{(b, s)}(k)+\left(1-\omega_{b}\right) v_{b} c_{n}^{(b, f)}(k)+\omega_{p} v_{p} c_{n}^{(p, s)}(k)+\left(1-\omega_{p}\right) v_{p} c_{n}^{(p, f)}(k)
$$

where, $c_{n}^{(\cdot,)}(k)$ indicates the solute concentration in the last mixing cell after the $k$-th drainage interval, and the indices $s, f, b, p$, denote the slow and fast domains (corresponding to matrix and macropore mixing cell models) and the bulk and plate regions, respectively. In addition, $\omega_{p}$ and $\omega_{b}$ are weighting factors for the plate and bulk regions, respectively, that weigh the contributions of their respective slow and fast domains. 
In our modelling approach, we start assuming equal average flow and transport characteristics in the bulk and the plate regions, i.e. $\omega_{p}=\omega_{b}=\omega, P e^{(b, s)}=P e^{(p, s)}, P e^{(b, f)}=P e^{(p, f)}, \theta^{(b, s)}=\theta^{(p, s)}$, and $\theta^{(b, f)}=\theta^{(p, f)}$. Therefore the number of parameters in the dual-domain, dual-region mixing-cell model is 5 . If additionally we assume $\omega_{p}=$ 1 and $\omega_{b}=1$, only the slow transport domains remain and the model is simplified to a single-domain, dualregion model with only 2 parameters $(P e, \theta)$. In contrast, the model complexity can be increased for both models by parameterizing the two transport regions separately $\left(P e^{(b \cdot)}, \theta^{(b \cdot)}, P e^{(p,)}, \theta^{p, \cdot)}\right)$.

\subsection{Parameter estimation and uncertainty assessment}

To estimate the mixing cell model parameters, we adopt two root-mean-square-error (RMSE) objective functions, $F_{1}$ and $F_{2}$, which jointly describe the quality of the fit to the $\mathrm{Br}^{-}$and $\mathrm{Cl}^{-}-\mathrm{BTCs}$ and the tracer outflow concentration as a function of cumulative drainage volume (abbreviated subsequently with CDV; essentially it is a BTC with drainage index). In a first step, the AMALGAM method (Vrugt \& Robinson, 2007) was used to analyse the trade-off between the two objective functions and the adequacy of the chosen model structure. AMALGAM combines two concepts, a simultaneous multimethod search and a self-adaptive offspring creation, to ensure a reliable and computationally efficient solution to multiobjective optimization problems. For further details on the method, please refer to Vrugt \& Robinson (2007).

In a second step, a Bayesian framework was adopted to derive parameter and model predictive uncertainty using the differential evolution adaptive metropolis, DREAM $\mathrm{ZS}_{\mathrm{ZS}}$ adaptive Markov chain Monte Carlo scheme and a formal likelihood function. We combine the data likelihood, $p(\mathbf{u} \mid \tilde{\mathbf{Y}})$, with a prior distribution $p(\mathbf{u})$ by Bayes theorem to infer the posterior probability density function of the model parameters:

$$
p(\mathbf{u} \mid \tilde{\mathbf{Y}}) \propto p(\tilde{\mathbf{Y}} \mid \mathbf{u}) p(\mathbf{u})
$$

If we assume the error residuals to be uncorrelated and normally distributed with constant variance, $\sigma^{2}$, the data likelihood function can be calculated as

$$
p(\mathbf{u} \mid \tilde{\mathbf{Y}})=\prod_{i=1}^{\mathrm{j}} \frac{1}{\sqrt{2 \pi \sigma^{2}}} \exp \left[-\frac{1}{2} \sigma^{-2}\left(y_{i}(\mathbf{u})-\widetilde{y}_{i}\right)^{2}\right]
$$

where $y_{i}(\mathbf{u})$ and $\tilde{y}_{i}, i=1, \ldots, j$ denote the model predicted values and their corresponding observations, respectively. In our approach, we prefer to use the log-likelihood function, $\ell(\mathbf{u} \mid \tilde{\mathbf{Y}})$, of (9) rather than $p(\mathbf{u} \mid \tilde{\mathbf{Y}})$ :

$$
\ell(\mathbf{u} \mid \tilde{\mathbf{Y}})=-\frac{j}{2} \ln (2 \pi)-\frac{j}{2} \ln \left(\sigma^{2}\right)-\frac{1}{2} \sigma^{-2} \sum_{i=1}^{j}\left(y_{i}(\mathbf{u})-\tilde{y}_{i}\right)^{2} .
$$

The prior distribution, $p(\mathbf{u})$ is assumed to be uniform with the following parameter ranges: $P e^{(;, s)}=[2 . .40]$; $P e^{(\cdot f)}=[40 . .80] ; \theta^{(s)}=[0.3 . .0 .7] ; \theta^{(f)}=[0 . .0 .1] ;$ and $\omega_{p}, \omega_{b}=[0 . .1]$. For more details on the methods, please refer to Wöhling \& Vrugt (2011) and Schoups and Vrugt (2010).

In our inverse modelling approach, we primarily focus on the assessment of parameter uncertainty. Measurement errors $\left(\sigma^{2}\right)$ are considered in (10) separately for solute concentration and drainage measurements. Other uncertainty sources that may contribute to the total model uncertainty, such as uncertainty in the model forcings (drainage intervals), other experimental errors, and model structural deficiencies are not explicitly treated here. Model uncertainties of the relatively simple mixing cell modelling approach adopted herein are caused for example by the assumption that preferential flow occurs at fixed proportions to matrix flow (steady weights for fast and slow flow paths as well as bulk and plate regions). However, model simplicity is beneficial when aiming at a quick hypothesis testing about the functioning of the flow and transport system under consideration.

The model parameters were estimated individually for AETL1 and AETL3 using observed $\mathrm{Cl}^{-}$BTCs and CDVs. Then the maximum likelihood (ML) parameter values were evaluated with simulations of the corresponding $\mathrm{Br}^{-}$BTCs and CDVs.

The fast solute transport components were negligible in AETL1. Therefore we adopted a one-domain, dual-region model $\left(\omega_{p}\right.$ and $\left.\omega_{b}=1\right)$ and estimated the corresponding parameters $(P e, \theta)$. Table 2. $\mathrm{Br}^{-}$and $\mathrm{Cl}^{-}$recovery of AETL 1 and AETL 3, and corresponding contributions of plate and bulk tracer.

\begin{tabular}{|l|l|l|l|l|}
\hline & $v_{p}$ & $v_{b}$ & \multicolumn{2}{|l|}{ Recovery [\%] } \\
\cline { 4 - 5 } & {$[\%]$} & {$[\%]$} & $\mathrm{Br}^{-}$ & $\mathrm{Cl}^{-}$ \\
\hline AETL1 & 54.8 & 44.3 & 54.9 & 97.5 \\
\hline AETL3 & 64.8 & 135.6 & 65.2 & 195.8 \\
\hline
\end{tabular}


Wöhling et al., Dual-domain mixing cell modelling and uncertainty analysis

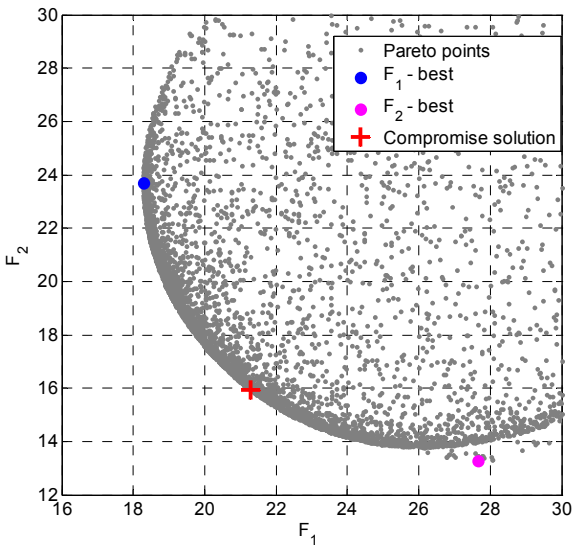

Figure 2. AMALGAM derived trade -off between $\mathrm{Cl}^{-}-\mathrm{BTC}\left(F_{1}\right)$ and $\mathrm{Cl}^{-}-\mathrm{CDV}\left(F_{2}\right)$ objective functions after 10,000 model evaluations of the AETL1 run. ing cell model with the parameters $P e^{(;, s)}, P e^{(; f f)}, \theta^{(; s)}, \theta$ (:f), $\omega_{p}$ and $\omega_{b}$.

\section{RESULTS}

\subsection{Quantifying tracer sources}

The $\mathrm{Br}^{-}$and $\mathrm{Cl}^{-}$concentrations in AETL3 and AETL1 peaked after 5 and 20 days, respectively, and receded to background levels after about 80 days of tracer application. The percentage of applied tracer volume from the plate and bulk areas, $v_{p}$ and $v_{b}$, was calculated by (4) and results are shown in Table 2. In the idealized case of vertical flow and transport (no lateral dispersion), we would obtain $v_{p}=100 \%$ and $v_{b}=0 \%$. However, the analysis reveals that only $54.8 \%$ of the plate tracer volume was captured by AETL1 and that $44.3 \%$ of the bulk tracer was collected (bulk tracer normalized by plate area). Note that the $\mathrm{Cl}^{-}$recovery appears to be quite high $(97.5 \%)$. This is a result of similar chloride concentration in both tracers and a total sampling volume of $99.1 \%$. It is unlikely that the low bromide recovery at AETL1 can be explained by dispersion alone. The maximum estimated alignment error $(20 \mathrm{~mm})$ between plate tracer application and the underlying AETL would only account for about $10 \%$ of the plate tracer loss. At the $0.4 \mathrm{~m}$ depth, however, the error from insufficient alignment is most likely smaller, since the AETL boundaries could be detected accurately by the GPR method. The discrepancy in bromide recovery is more likely caused by unsteady flow and lateral dispersion in the vadose zone.

In case of AETL3, an equivalent volume of $135.6 \%$ originates from the bulk region which results in a very large $\mathrm{Cl}^{-}$recovery (Table 2). The large sampling volume is also an indication for preferential flow. This was confirmed Table 3. Parameter estimates and objective function values for the AETL1 single-domain mixing cell model.

\begin{tabular}{|l|l|l|c|c|}
\hline & $\theta$ & $P e$ & $F_{1}[\mathrm{mg} / 1]$ & $F_{2}[\mathrm{mg} / 1]$ \\
\hline AMALGAM - best $F_{1}$ & 0.37 & 14.5 & 18.3 & 56.6 \\
\hline AMALGAM - best $F_{2}$ & 0.31 & 10.6 & 60.7 & 14.8 \\
\hline AMALGAM - compromise & 0.33 & 12.1 & 35.8 & 30.0 \\
\hline DREAM, max. likelihood & 0.34 & 12.7 & 30.8 & 30.4 \\
\hline
\end{tabular}
by the early peak in the BTC (see below).

\subsection{Parameter estimation and Trade-off analysis}

Results for the AETL 1 - AMALGAM run are depicted in Figure 2 which shows the non-dominated, or Pareto solutions that define the trade-off between the $\mathrm{Cl}^{-}-\mathrm{BTC}\left(F_{1}\right)$ and $\mathrm{Cl}^{-}-\mathrm{CDV}\left(F_{2}\right)$ objective functions. The curved shape of the Pareto front shows that no parameter set simultaneously satisfies both objective functions equally well. A compromise solution (red + ) was selected that minimizes $F_{1}+F_{2}$. The parameters of the best attainable objective function values of $F_{1}$ and $F_{2}$ (blue and magenta), and the compromise solution are listed in Table 3 and Table 4 for the AETL1 and AETL3 models, respectively. The large trade-off between the objective functions can be explained by measurement errors of experimental data, but also by structural inadequacies of the adopted mixing cell modeling approach (c.f. section 2.4.). A separate parameterization of the bulk and plate regions in the AETL1 mixing cell model did not result in a significant reduction of the tradeoff between the objective functions or in an improvement of the objective function values.

\subsection{Uncertainty assessment and model evaluation}

The posterior probability density functions of parameter and total model uncertainty were calculated for AETL1 and AETL3 by the DREAM 95\% parameter and total model uncertainty bounds are shown in the upper panels of Figures 3 and 4. The ML parameter values are listed in Tables 3 and 4 . The fits to the $F_{1}$ and $F_{2}$ objective functions are similar or

Table 4. Parameter estimates and objective function values for the AETL3 dual-domain mixing cell model.

\begin{tabular}{|l|l|l|l|l|l|c|c|}
\hline & $\theta^{(s)}$ & $\theta^{(f)}$ & $P e^{(; s)}$ & $P e^{(j, f)}$ & $\omega$ & $F_{1}[\mathrm{mg} / \mathrm{l}]$ & $F_{2}[\mathrm{mg} / \mathrm{l}]$ \\
\hline AMALGAM - best $F_{1}$ & 0.34 & 0.076 & 7.54 & 62.6 & 0.427 & 19.8 & 25.8 \\
\hline AMALGAM - best $F_{2}$ & 0.34 & 0.081 & 6.96 & 51.1 & 0.460 & 28.9 & 14.9 \\
\hline AMALGAM - compromise & 0.33 & 0.079 & 7.07 & 51.5 & 0.444 & 23.0 & 17.3 \\
\hline DREAM, max. likelihood & 0.35 & 0.084 & 7.52 & 77.8 & 0.444 & 20.6 & 16.7 \\
\hline
\end{tabular}


better (lower RMSE values) than the values of their corresponding AMALGAM compromise solutions.

The observed BTC of AETL1 is unimodal which indicates that the solute transport is dominated by a single transport domain. The average width of the parameter uncertainty bounds is very small $(0.022 / 0.021 \mathrm{mg} / 1 \mathrm{for}$ the $\mathrm{Cl}^{-} \mathrm{BTC} / \mathrm{CDV}$ ) because the model employs only 2 parameters. The average width of the total uncertainty bound was $127.3 / 117.0 \mathrm{mg} / \mathrm{l}$ for the $\mathrm{Cl}^{-} \mathrm{BTC} / \mathrm{CDV}$. Simulations with the ML parameter values generally fit well to the observed data which was confirmed by relatively large $\mathrm{R}^{2}$ - values $(0.94 / 0.95)$. The evaluation of the $\mathrm{Br}^{-} \mathrm{BTC} / \mathrm{CDV}$ as simulated with the ML parameter values resulted in somewhat lower $\mathrm{R}^{2}$ values $(0.87 / 0.81)$ because the peak concentrations are underestimated (Figure 3$)$. This resulted also in a slightly lower coverage of the observations by the model uncertainty bounds $(90.0 / 86.7 \%)$.
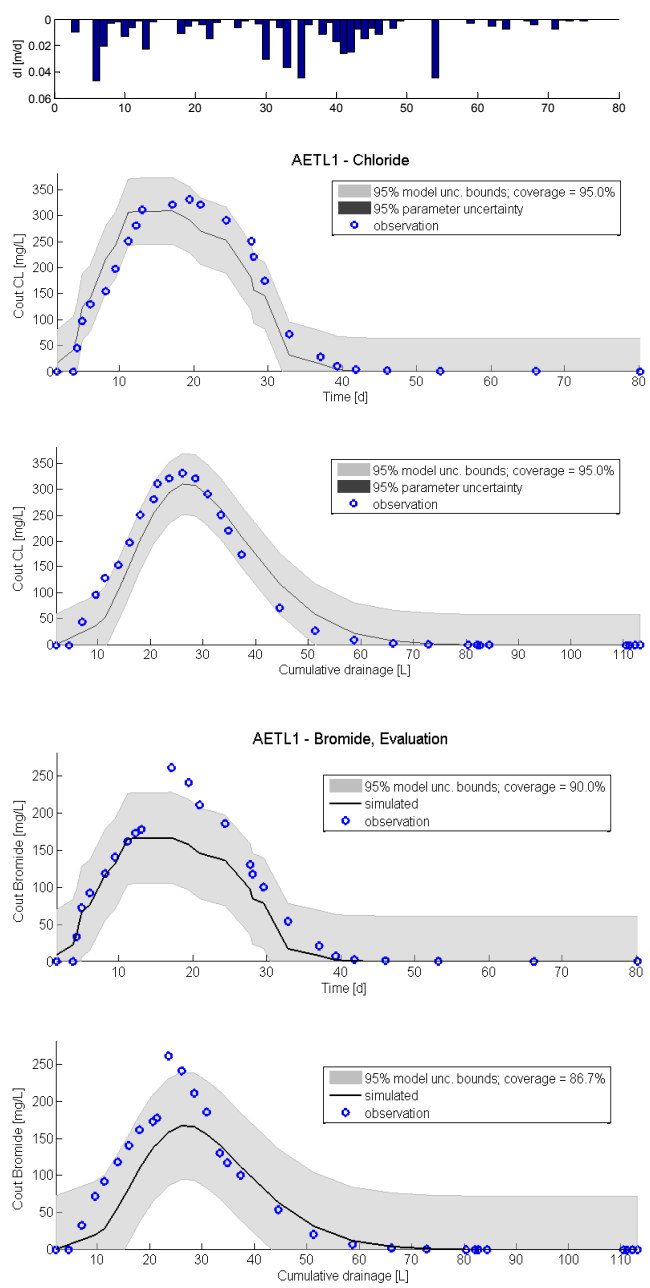

Figure 3. Observations (blue) and simulated 95\% parameter and model uncertainty bounds for breaktrough curves of AETL1. The panels show drainage intervals (top), the model calibration $\left(\mathrm{Cl}^{-}\right.$; middle), and model evaluation ( $\mathrm{Br}^{-}$; bottom), respectively.
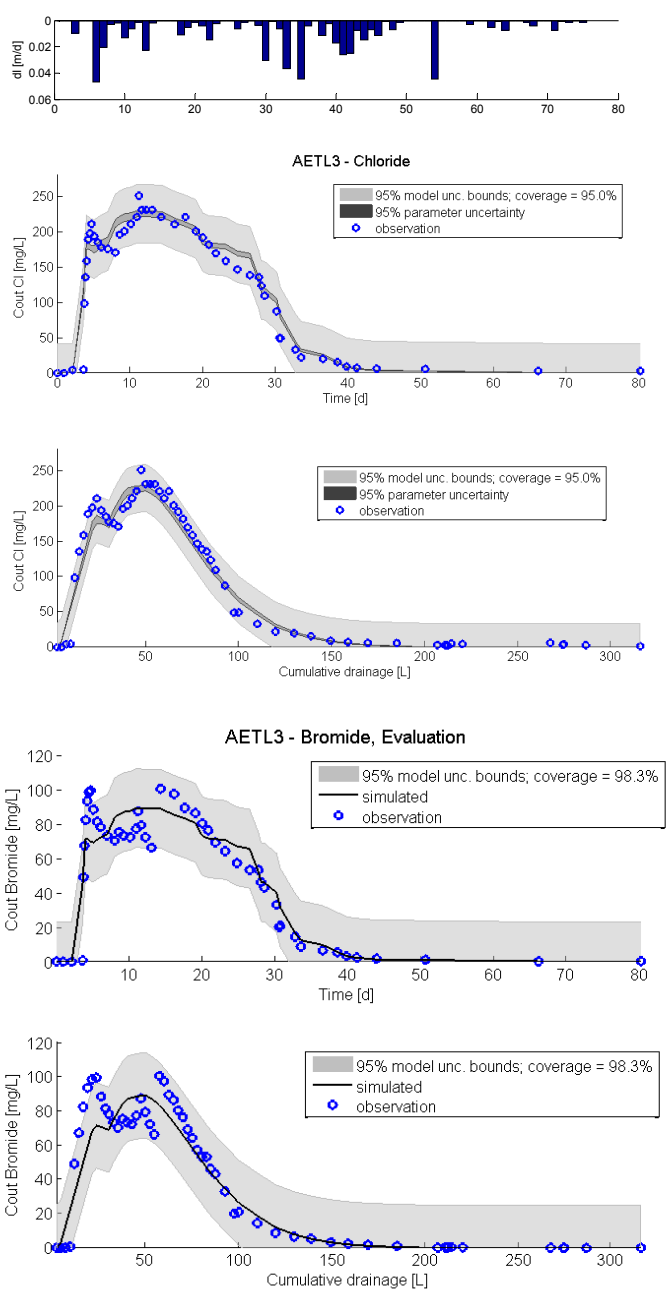

Figure 4. Observations (blue) and simulated 95\% parameter and model uncertainty bounds for breaktrough curves of AETL3. The panels show drainage intervals (top), the model calibration $\left(\mathrm{Cl}^{-}\right.$; middle), and model evaluation ( $\mathrm{Br}^{-}$; bottom), respectively.

In contrast to AETL1, the BTC of AETL3 is bimodal with a distinguished early peak related to preferential flow components and a delayed second peak related to a slower component with a similar timing as the peak observed in AETL1 (Figures 3 and 4). The average width of the 95\% parameter and total model uncertainty bounds is $5.6 / 5.4 \mathrm{mg} / 1$ and $82.6 / 66.6 \mathrm{mg} / 1$ for the $\mathrm{Cl}^{-} \mathrm{BTC} / \mathrm{CDV}$, respectively. The total uncertainty bounds are smaller than for AETL1 and cover the bimodal observations well. The simulation with the ML parameter values matches the data which was confirmed by large $\mathrm{R}^{2}$ - values $(0.95 / 0.97)$. The $\mathrm{Br}^{-} \mathrm{BTC}$ and CDV were evaluated with the ML parameter set (Figure 4, lower panels). The bromide concentration peaks are not exactly matched by the simulation but the coverage of the $95 \%$ model uncertainty bounds is quite large $(92.3 / 92.3 \%)$ and the average fit to the data is satisfactory $\left(\mathrm{R}^{2}=0.91 / 0.89\right)$. 
The maximum likelihood parameter estimation revealed that the transporting volume fraction in the matrix domain is very similar for AETL1 and AETL3 (0.34 and 0.35) which is in agreement with the relative homogeneity of the vadose zone texture. However, the transporting volume fraction is only about half of the total porosity (66 - 68\%; c.f. Wöhling et al. 2008). Dispersivity in this domain is somewhat larger for AETL3 as compared to AETL1 (Tables 3 and 4). Our results show further that preferential flow in AETL3 occurs in less than $1 \%$ of the volume fraction.

\section{CONCLUSIONS}

The multiple tracer experiment at the Spydia site revealed highly variable flow and transport in the shallow vadose zone. This large variability was not necessarily expected for the relatively homogeneous textured Ignimbrite materials. Although AETL1 appears to be unaffected by preferential flow paths, only 55\% of the solute originating from the area directly above the lysimeter was captured. In contrast, preferential flow occurring in AETL3 resulted in a characteristic bimodal breakthrough curve and large drainage volumes. The preferential solute transport most likely originates from areas not directly above the plate. The equivalent of $136 \%$ of the applied volume was captured by AETL3 from the bulk region and only $65 \%$ from the region directly above the lysimeter.

Our results suggest that even in the homogeneous volcanic materials contaminants from the land surface can pass the soil zone relatively quickly and thus can potentially reach larger depths (and the groundwater) quite fast. Unsteady flow and lateral flow components in the matrix and on stratigraphic boundaries may have contributed in channelling the transport. The dual-domain mixing cell model proved to be useful for simulating advective-dispersive transport in a fast and a slow transport domain and two regions at the soil surface that were marked with different tracer compositions. The model simulations using the maximum likelihood parameter values and the $95 \%$ total uncertainty bounds compare well to the measured $\mathrm{Br}^{-} / \mathrm{Cl}^{-}$data. The mixing cell model used herein is an efficient tool for analysing single and multiple tracer experiments. It contributes to a better understanding of contaminant pathways on their way from the land surface through the subsurface to rivers and lakes.

\section{ACKNOWLEDGMENTS}

This work was funded under the New Zealand Foundation for Research, Science and Technology "Groundwater Quality" (LVLX0302) and "Groundwater Assimilative Capacity" (C03X1001) contracts.

\section{REFERENCES}

Barkle, G.F., Wöhling, Th., Stenger, R., Mertens, J., Moorhead, B., Wall, A. and Claque, J. (2011). Measuring water and contaminants fluxes throughout the vadose zone using Automated Equilibrium Tension Lysimeters (AETLs). Vadose Zone Journal, 10(2), 747-759.

Bidwell, V. J. (1999). A formula for predicting advection-dispersion in the vadose zone at uneven drainage intervals. Water Resources Research, 36(10), 3077-3080.

Bidwell, V.J. (2000). State-space mixing cell model of unsteady solute transport in unsaturated soil. Environmental Modelling \& Software, 14, 161-169.

Dann, R., Bidwell, V., Thomas, S., Wöhling, Th., Close, M. (2010). Modelling of Nonequilibrium Bromide Transport through Alluvial Gravel Vadose Zones. Vadose Zone Journal, 9, 731-746.

MfE (2007). State of the Environment report. Ministry for the Environment, PO Box 10362, Wellington, New Zealand, publication number ME 847, ISBN: 978-0-478-30191-5.

Schoups, G.H., Vrugt, J.A. (2010). A formal likelihood function for parameter and predictive inference of hydrologic models with correlated, heteroscedastic, and non-Gaussian errors. Water Resources Research, 46, W10531.

Vrugt, J.A. \& Robinson, B. A. (2007). Improved evolutionary optimization from genetically adaptive multimethod search. Proc. of the Nat. Academy of Sci. of the United States of America (PNAS), 104, 708-711.

Vrugt, J.A., Laloy, E., ter Braak, C.J.F. (2011). Differential evolution adaptive Metropolis with sampling from past states. SIAM Journal on Optimization. In review.

Wöhling, Th., Barkle, G.F., Vrugt, J.A. (2008). Comparison of three multiobjective optimization algorithms for inverse modeling of vadose zone hydraulic properties. Soil Sci. Soc. of America J., 72(2), 305-319.

Wöhling, Th., Schütze, N., Heinrich, B., Šimunek, J. and Barkle, G.F. (2009). Three-dimensional modeling of multiple Automated Equilibrium Tension Lysimeters to measure vadose zone fluxes. Vadose Zone Journal, 8(4), 1051-1063.

Wöhling, Th., Vrugt, J.A. (2011). Multi-response multi-layer vadose zone model calibration using Markov chain Monte Carlo simulation and field water retention data. Water Resources Research, 47, W04510. 